\title{
Quantitative evaluation of brain volume among elderly individuals in São Paulo, Brazil: a population-based study
}

Avaliação quantitativa do volume cerebral em um grupo de idosos de São Paulo, Brasil: um estudo de base populacional

Mariana Athaniel Silva Rodrigues ${ }^{1, a}$, Thiago Pereira Rodrigues ${ }^{2, b}$, Mayana Zatz ${ }^{3, c}$, Maria Lúcia Lebrão ${ }^{4, \dagger}$, Yeda Aparecida Duarte $^{5, d}$, Michel Satya Naslavsky ${ }^{3, e}$, Felipe Barjud Pereira do Nascimento ${ }^{1, f}$, Edson Amaro Junior ${ }^{1, g}$

1. Department of Radiology and Diagnostic Imaging, Hospital Israelita Albert Einstein, São Paulo, SP, Brazil. 2. Escola Paulista de Medicina da Universidade Federal de São Paulo (EPM-Unifesp), São Paulo, SP, Brazil. 3. Biosciences Institute, Universidade de São Paulo, São Paulo, SP, Brazil. 4. School of Public Health, Universidade de São Paulo, São Paulo, SP, Brazil. 5. School of Nursing, Universidade de São Paulo, São Paulo, SP, Brazil.

Correspondence: Dra. Mariana Athaniel Silva Rodrigues. Hospital Israelita Albert Einstein - Departamento de Radiologia e Diagnóstico por Imagem. Avenida Albert Einstein, 627, Jardim Leonor. São Paulo, SP, Brazil, 05652-900. Email: mariathaniel@gmail.com.

a. http://orcid.org/0000-0001-7566-3632; b. https://orcid.org/0000-0002-4643-5339; c. https://orcid.org/0000-0003-3970-8025; d. https://orcid.org/0000-0003-3933-2179; e. https://orcid.org/0000-0002-9068-1713; f. https://orcid.org/0000-0003-4319-3386; g. https://orcid.org/0000-0002-5889-1382.

Received 25 June 2018. Accepted after revision 11 January 2019.

† In memoriam.

How to cite this article:

Rodrigues MAS, Rodrigues TP, Zatz M, Lebrão ML, Duarte YA, Naslavsky MS, Nascimento FBP, Amaro Junior E. Quantitative evaluation of brain volume among elderly individuals in São Paulo, Brazil: a population-based study. Radiol Bras. 2019 Set/Out;52(5):293-298.

Abstract Objective: To perform a quantitative analysis of the brain volume of elderly individuals in a population-based sample.

Materials and Methods: This was a radiological assessment and voxel-based quantitative analysis, with surface alignment, of 525 magnetic resonance imaging scans of individuals between 60 and 103 years of age who participated in the Saúde, Bem-estar e Envelhecimento (Health, Well-being, and Aging) study in the city of São Paulo, Brazil.

Results: We noted a median rate of reduction in total brain volume of $2.4 \%$ per decade after 60 years of age. Gray and white matter both showed volume reductions with age. The total brain volume/intracranial brain volume ratio differed between males and females.

Conclusion: We have corroborated the findings of studies conducted in the United States and Europe. The total brain volume/intracranial brain volume ratio is higher in men, representing a potential bias for the conventional radiological assessment of atrophy, which is typically based on the evaluation of the cerebrospinal fluid spaces.

Keywords: Aging; Brain; Magnetic resonance imaging; Neuroimaging; Image interpretation, computer-assisted/methods.

Resumo Objetivo: Analisar, quantitativamente, o volume cerebral de idosos em uma amostra de base populacional em São Paulo.

Materiais e Métodos: 0 estudo é uma avaliação radiológica e análise quantitativa baseada em voxel com alinhamento de superfície de 525 imagens de ressonância magnética de participantes de uma coorte de idosos (SABE - Saúde, Bem-estar e Envelhecimento) em São Paulo, Brasil, com idades de 60 a 103 anos, dos dois sexos.

Resultados: Observamos redução média do volume cerebral total de 2,4\% por década após os 60 anos de idade. A redução de volume ocorreu na substância cinzenta e na substância branca com a idade. A relação entre volume cerebral intracraniano e volume cerebral total diferiu entre homens e mulheres.

Conclusão: Nós reproduzimos os achados de estudos prévios em populações americanas e europeias. A relação entre volume intracraniano e volume cerebral é maior em homens, o que pode representar fonte de viés na avaliação de atrofia radiológica convencional, já que essa avaliação é usualmente baseada em análise de espaços liquóricos intracranianos.

Unitermos: Envelhecimento; Encéfalo; Ressonância magnética; Neuroimagem; Interpretação de imagem assistida por computador/ método.

\section{INTRODUCTION}

Normal aging is associated with gradual brain atrophy ${ }^{(1)}$. Almost all of the studies conducted to date, regardless of the methodology employed, have confirmed the basic observation that as adults age, their brains become smaller and the sulci increase in size and depth ${ }^{(2)}$. However, cerebral atrophy is a complex phenomena that is only partially related to a reduction in the number of neurons, which varies less than $10 \%$ from 20 to 90 years of age; it also occurs as a consequence of myelin loss-apparently preceding the neuronal loss ${ }^{(3)}$. Although the neocortex shows milder changes, neuron loss in other areas, the hippocampus in particular, remains quite stable over a lifetime ${ }^{(4)}$. Other factors, including intercellular space 
loss, water reduction, and vascular changes, may also play a role in the reduction in brain volume during the normal aging process ${ }^{(5)}$.

The use of high-resolution magnetic resonance imaging (MRI) has enabled the detailed assessment of agingrelated changes in brain morphometry ${ }^{(6)}$. Although estimates typically indicate that brain tissue volume is smaller in older adults than in younger individuals, data regarding the effects of aging on gray matter versus white matter are not consistent ${ }^{(7)}$ and continue to constitute a topic of investigation. The contradictory findings might be attributable to the relatively small sample sizes. Most studies of the topic have analyzed samples comprising $\leq 200$ subjects-Allen et al. ${ }^{(8)}$ included 87 subjects, Giorgio et al. ${ }^{(1)}$ included 66 subjects, and Raz et al. ${ }^{(2)}$ included 200 participants - and have not recruited subjects using epidemiological sampling. Another technical aspect that could result in variations across studies is the method of analysis, which rarely takes factors such as race and ethnicity into account. Therefore, normalization of morphometric analyses by intracranial volume (ICV) is used in order to correct the head size data and is believed to be fundamental to reducing false statistical interpretation of brain volume and provides the basis for between-patient comparisons ${ }^{(9)}$.

The recent literature on dementia is densely populated by quantitative MRI studies ${ }^{(10)}$. It is not only relevant to measure overall brain atrophy but also useful to describe regional reductions in brain volume. For instance, atrophy of the hippocampus has been found to correlate strongly with the severity of Alzheimer's disease, as determined by pathology at autopsy. Accurate quantification of the region-specific effects of aging is important for establishing a structural paradigm to identify age-related regional cortical changes and disease-specific regional cortical volume changes such as those that occur in Alzheimer's disease $^{(11)}$. However, in the clinical setting, quantitative volumetric analysis of the brain is not always possible, because of financial constraints (especially in public health care systems), poor MRI technique, or limited operator knowledge for quality evaluation of the automated results. In this context, most radiology reports are based only on subjective assessment of intracranial structures. In addition, the majority of medical residency training programs have yet to incorporate quantitative biomarker evaluation methods. Most of the training is conventional, mostly reflecting self-experience based on individual learning and exposure to relevant cases.

There have been attempts to improve the visual assessment of brain MRI via the use of predefined atrophy scores, as a means of reducing interindividual variation. Examples include the visual rating system for medial temporal atrophy and the entorhinal cortex atrophy score $^{(10,12)}$. The overall severity of atrophy in the medial temporal lobe, as well as in the hippocampus, entorhinal cortex, and perirhinal cortex, can be scored with the visual rating system for medial temporal atrophy, as proposed by Shen et al. ${ }^{(13)}$, which apparently has better discriminatory power than do volumetric methods and shows a strong correlation with memory scores. However, these scores can be calculated only from coronal images intersecting the mammillary bodies, which require correct image plane acquisition, a condition that is not always met in clinical settings.

The purpose of this epidemiological MRI study was to describe the profile of elderly individuals living in a megacity in South America, in terms of brain volume, by age and gender; to compare our findings with those of similar studies conducted in other regions of the world; and to describe the MRI appearance of the median-volume brains in each age group. We believe that this approach could inform future quantitative volumetric analyses, as well as helping clinical radiologists by providing normative information and serving as a proxy for an atlas to support daily practice.

\section{MATERIALS AND METHODS}

Volunteers were recruited, through the use of an epidemiological sampling method, in the city of São Paulo, Brazil, throughout the year of 2013. All participants gave written informed consent. Of the 525 volunteers participating on this studied, $331(63.0 \%)$ were women. The distribution of the participants by age group was as follows: 60-64 years, 89 (17.0\%); 65-69 years, $118(22.5 \%)$; 70-74 years, $79(15.0 \%)$; 75-79 years, 70 (13.3\%); 80-84 years, 78 (14.8\%); 85-89 years, 59 (11.2\%); 90-94 years, $25(4.8 \%)$; and $95-103$ years, $7(1.3 \%)$.

All MRI examinations were performed in the same 3.0 T scanner (Tim Trio; Siemens Healthcare, Erlangen, Germany), with a 32-channel head coil, and the same examination protocol. A T1-weighted volumetric sequence, based on the Alzheimer's Disease Neuroimaging Initiative 2 protocol $^{(14)}$, was acquired with the following parameters: T1-weighted, three-dimensional magnetization-prepared rapid-acquisition gradient echo imaging; 1-mm isotropic voxels; repetition time: $2500 \mathrm{~ms}$; echo time: $3.45 \mathrm{~ms}$; inversion time: $1100 \mathrm{~ms}$; and flip angle: $7^{\circ}$. Four trained neuroradiologists analyzed the images to exclude visible abnormalities and head movement artifacts.

Images were processed and analyzed with FreeSurfer software, version 5.3.0 (Athinoula A. Martinos Center for Biomedical Imaging, Massachusetts General Hospital, Boston, MA, USA), on a high-performance computer, to construct models of the cortical surface. These wellvalidated and fully automated procedures were described in detail by Fischl et al. ${ }^{(15)}$. In brief, after intensity normalization, skull stripping, and image segmentation, we generated a single filled white-matter volume was for each hemisphere, using a connected component algorithm. We then generated a surface tessellation for each white-matter volume by fitting a deformable template. This process 
resulted in a triangular cortical mesh for the gray- and white-matter surfaces consisting of approximately 150,000 vertices (i.e., points) per hemisphere. Measures of cortical thickness were computed as the closest distance from the gray- and white-matter boundaries to the gray matter and cerebrospinal fluid boundary at each vertex on the tessellated surface. Thickness data were smoothed using a 10-mm surface-based smoothing kernel. The quantitative results were grouped into age groups of 5 years each and were compared by gender.

\section{RESULTS}

As expected, we noted a reduction in brain volume in the older age groups (Figure 1). The median rate of brain volume reduction was $2.4 \%$ per decade after 60 years of age. Among the 5-year intervals (from 60 to 105 years), the rate varied from $0.2 \%$ to $4.6 \%$, although the differences were not statistically significant. We also observed no significant difference between the gray and white matter in terms of the rate of volumetric reduction (Figure 2).

In each of the age groups studied, the total (uncorrected) brain volume was higher in the men than in the women (Figure 1). However, the ICV-corrected brain volumes were higher in the women (Figure 3 ).

Qualitative analysis (visual assessment) of the brain images showed enlargement of the intracranial cerebrospinal fluid spaces with aging. Sulci enlargement was consistent with aging and was more related to the age groups than to the changes in ventricular size (Figure 3).

\section{DISCUSSION}

Aging-related reductions in overall brain volume have been widely explored in the literature and have been observed for gray and white matter alike. Walhovd et al. ${ }^{(16)}$ assessed the heterogeneous age responses of various brain volumes and found that age led to a decline in

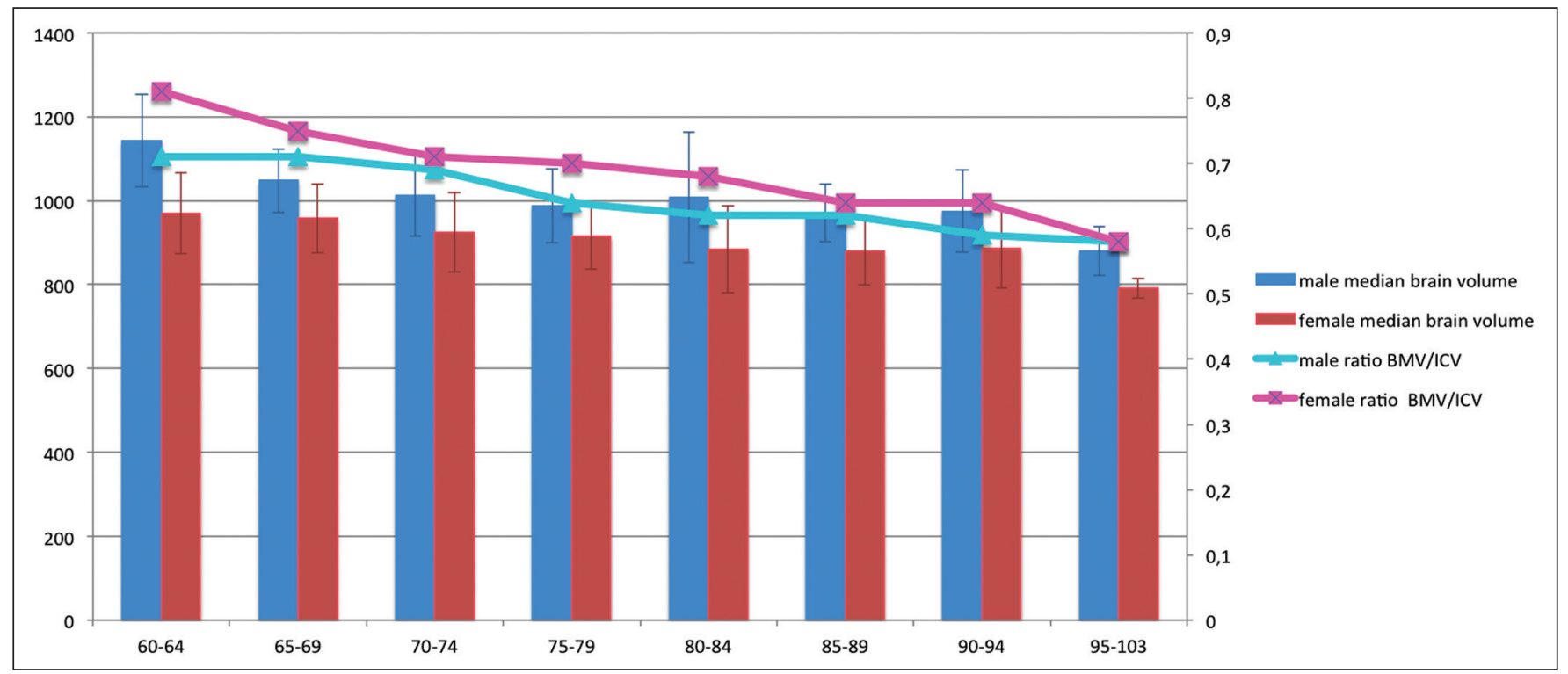

Figure 1. Brain volume, ICV/total brain volume ratio, and standard deviation, by age group and gender.

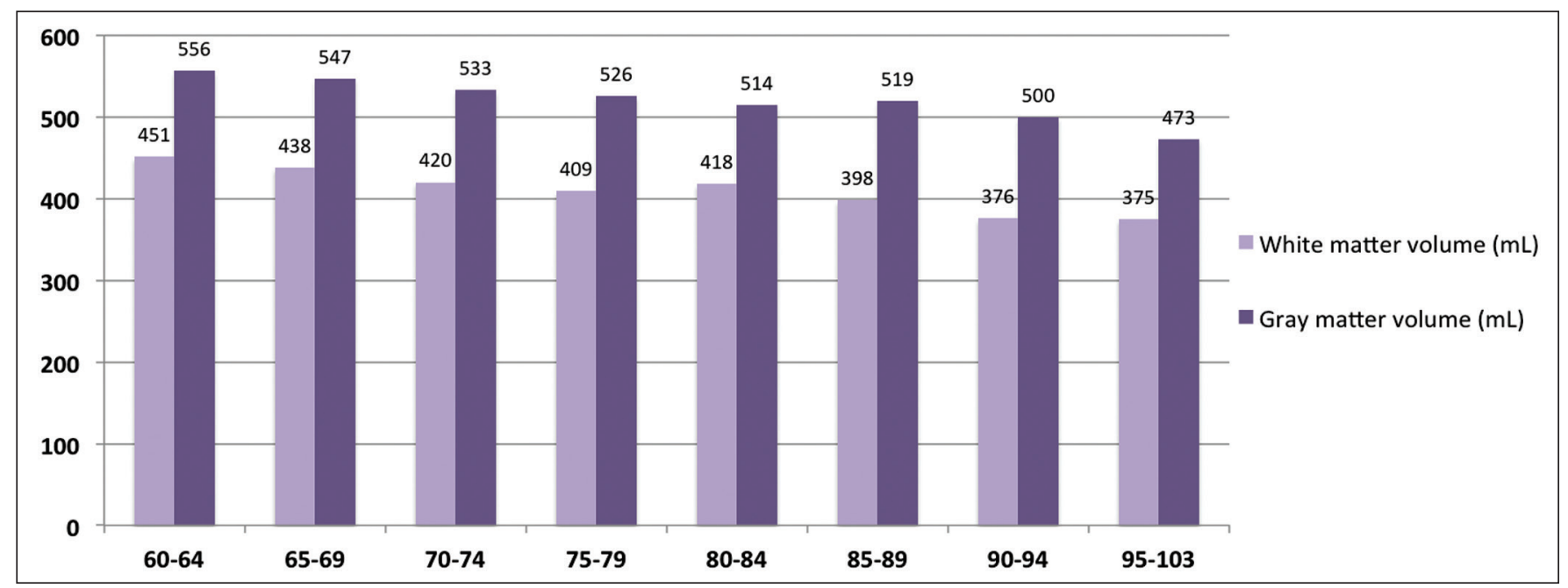

Figure 2. Gray- and white-matter volume distribution, by age group. 


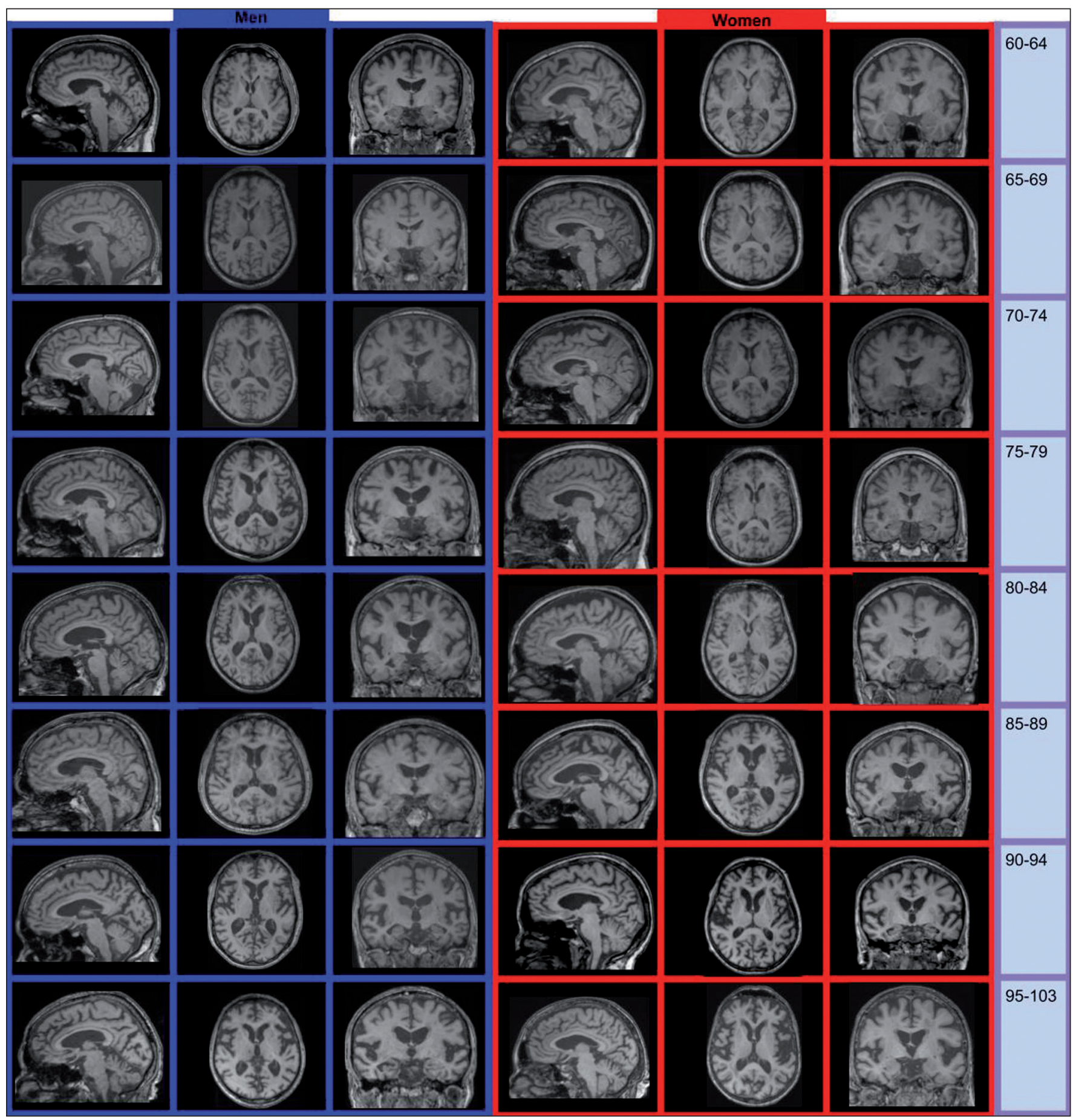

Figure 3. MRI scans of median-volume brains, by age group.

white-matter structures, as well as in all gray-matter and subcortical structures, with the exception of the globus pallidus. Conversely, Giorgio et al. ${ }^{(1)}$ reported specific brain areas for which they found linear or nonlinear correlations between volume and age. In the present study, we evaluated the entire brain, assessed the white- and gray-matter volumes independently. The qualitative evaluation of the entire brain remains accepted as an important first impression and plays an important role in clinical practice.
A population-based approach to recruiting the participants in our study likely yielded results different from those obtained when convenience samples are employed ${ }^{(6)}$. In a one-year longitudinal study, Resnick et al. ${ }^{(7)}$ studied the ventricle-brain ratio and found consistent data, revealing a small but significant increase over the study period. In the present cross-sectional study, we assessed ventricle size by age group but could not prove that it increases with age, the visual analysis showing that the size of the sulci increased more consistently. 
In the present study, the total (uncorrected) brain volumes were typically larger in the men than in the women, in all of the age groups studied, whereas the ICV-corrected brain volumes were lower in the men. This result is in line with those of the majority of studies. For instance, Resnick et al. ${ }^{(7)}$ also found higher uncorrected brain volumes in men. However, the authors did not find any changes when brain volume was corrected for height. This last finding is in apparent contrast to those of our study. However, we used a different type of volumetric acquisition and evaluated data obtained through voxel-based volumetry (with and without ICV correction). In fact, when we compared our results with those of Salgorzaei et al. ${ }^{(9)}$, there were even more similarities. Those authors found that the values for brain volume and ICV were higher in women than in men. In addition, the median rate of reduction in brain volume over time in the present study was approximately $2.4 \%$ per decade after age 60 , which is comparable to the rates reported in previous studies. Miller et al. ${ }^{(17)}$ posited that the rate of volume loss in the brain as a whole is $2 \%$ per decade, similar to the approximately $1.9 \%$ posited by Seshadri et al. ${ }^{(18)}$. As previously mentioned, the rates observed for the various age groups evaluated in the present study ranged from $0.2 \%$ to $4.6 \%$. That variation may reflect the population-based nature of our evaluation, the MRI parameters evaluated, or simply the analysis methods employed. Radiological interpretation based on visual assessment, even when a comprehensive rating scale is used, has a higher rate of interobserver variation than does the evaluation of age-related brain volume reduction ${ }^{(19)}$. We suspect that the overall effect observed for the median rate of total (uncorrected) brain volume reduction is not sufficient to be detected by conventional (non-quantitative) radiological evaluation in clinical practice.

Another important aspect of our study is the epidemiological sampling method. Most studies of this topic employ conventional recruiting methods. Volunteers respond to announcements or are part of the hard-to-reach populations. However, the selection of "super-normal" volunteers as control subjects in investigations of disease mechanisms, especially those related to the aging process, does not constitute a valid means of creating a group of controls that are representative of the typical patients seen in clinical practice. The selection of obviously "successfully aging subjects" is not equivalent to that of "asymptomatic" healthy individuals and constitutes a potential source of bias $^{(8)}$. Our data sample was derived from the Saude, Bemestar e Envelhecimento (Health, Well-being, and Aging) study $^{(20)}$, funded by the Pan American Health Organization and conducted in major cities in South America, which was designed to provide the best possible representation of the target population. Population-based study designs are likely to reduce selection biases by ensuring that all individuals enrolled in the study have levels of exposure to environmental factors similar to those of the general population ${ }^{(6)}$.
Considering the median brain volume loss in a more comprehensive scenario helps extrapolate these results to daily practice, because the neuropsychological history is usually incomplete as well as because there are a substantial number of conditions, including vascular diseases, dementia, medications, traumas, and infectious diseases, of varying intensities, all acting together.

One previous study found good agreement between a radiologist and neurosurgeons in terms of the overall interpretation of computed tomography scans of the brain, although the level of agreement was found to be poor for assessments of leukoaraiosis and reduced brain volume ${ }^{(21)}$. Subtle MRI findings tend to result in poor inter-rater reliability, although there are divergent results in the literature regarding the exact degree of inter-rater reliability for each finding or medical specialty. That underscores the need for automated methods of obtaining quantitative data on brain volume loss-especially given the rapidly evolving aging process among city dwellers in South America. We believe that an approach such as the one we have adopted herein may help improve understanding of this phenomena.

Specifically in routine radiological practice, normative imaging data are an important source of comparison, such data also being used with regularity in residency and subspecialty training programs. Although the images presented in our study were not acquired to that end, they provide a source of visual comparison that could be useful in cases of inconclusive findings regarding volumetric changes in the brain, especially when quantitative data are not available.

One interesting finding of the present study is that the ratio between the ICV and total brain volume differed between men and women. The men tended to have a smaller brain volume relative to the ICV than did the women. A possible consequence is that reports of MRI brain evaluations might be more likely to mention atrophy (or related terms) when the subject is male, given that the radiological interpretation is comparative; that is, if the enlarged cerebrospinal fluid spaces are visually more prominent (as they are in men), the degree of brain reduction might be overestimated.

\section{CONCLUSION}

The determination of brain volume is an important tool for radiologists in clinical practice. The comparison with peers from the same region, gender, and age group is crucial. Previous studies have reported that men have bigger brains than do women, although the ratio of brain matter to ICV is higher in women. The rate of brain volume reduction observed in our sample is in agreement with that reported in previous studies. The MRI scans of the median-volume brains, by age group, presented in this population-based study could be helpful in clinical practice. There is a need for further studies, involving a more in-depth investigation of this topic, in order to promote 
earlier, more accurate diagnosis of a wide variety of diseases that are prevalent among the elderly.

\section{REFERENCES}

1. Giorgio A, Santelli L, Tomassini V, et. al. Age-related changes in grey and white matter structure throughout adulthood. Neuroimage. 2010;51:943-51.

2. Raz N, Gunning-Dixon F, Head D, et al. Aging, sexual dimorphism, and hemispheric asymmetry of the cerebral cortex: replicability of regional differences in volume. Neurobiology of Aging. 2004;25:377-96.

3. Pakkenberg B, Pelvig D, Marner L, et al. Aging and the human neocortex. Exp Gerontol. 2003;38:95-9.

4. Andersen BB, Gundersen HJ, Pakkenberg B. Aging of the human cerebellum: a stereological study. J Comp Neurol. 2003;466:356-65.

5. Oliveira-Pinto AV, Andrade-Moraes CH, Oliveira LM, et al. Do age and sex impact on the absolute cell numbers of human cerebral regions? Brain Struct Funct. 2016;221:3547-59.

6. Terribilli D, Schaufelberger MS, Duran FLS, et al. Age-related gray matter volume changes in the brain during non-elderly adulthood. Neurobiol Aging. 2011;32:354-68.

7. Resnick SM, Goldszal AF, Davatzikos C, et al. One-year age changes in MRI brain volumes in older adults. Cereb Cortex. 2000;10:46472.

8. Allen JS, Bruss J, Brown CK, et al. Normal neuroanatomical variation due to age: the major lobes and a parcellation of the temporal region. Neurobiol Aging. 2005;26:1245-60.

9. Sargolzaei S, Sargolzaei A, Cabrerizo M, et al. A practical guideline for intracranial volume estimation in patients with Alzheimer's disease. BMC Bioinformatics. 2015;16 Suppl 7:S8.

10. Frisoni GB, Fox NC, Jack CR Jr, et al. The clinical use of structural MRI in Alzheimer disease. Nat Rev Neurol. 2010;6:67-77.

11. Peng F, Wang L, Geng Z, et al. A cross-sectional voxel-based morphometric study of age- and sex-related changes in gray matter volume in the normal aging brain. J Comput Assist Tomogr. 2016; 40:307-15.

\section{$(\boldsymbol{C c})$ BY}

12. Enkirch SJ, Traschütz A, Müller A, et al. The ERICA score: an MR imaging-based visual scoring system for the assessment of entorhinal cortex atrophy in Alzheimer disease. Radiology. 2018;288:22633.

13. Shen Q, Loewenstein DA, Potter E, et al. Volumetric and visual rating of magnetic resonance imaging scans in the diagnosis of amnestic mild cognitive impairement and Alzheimer's disease. Alzheimers Dement. 2011;7:e101-e108.

14. Jack CR Jr, Bernstein MA, Borowski BJ, et al. Update on magnetic resonance imaging corte of the Alzheimer's disease neuroimaging initiative. Alzheimers Dement. 2010;6:212-20.

15. Fischl B, Dale AM. Measuring the thickness of the human cerebral cortex from magnetic resonance images. Proc Natl Acad Sci USA. 2000;97:11050-5.

16. Walhovd KB, Fjell AM, Reinvang I, et al. Effects of age on volumes of cortex, white matter and subcortical structures. Neurobiol Aging. 2005;26:1261-70.

17. Miller AKH, Alston RL, Corsellis JAN. Variation with age in the volumes of grey and white matter in the cerebral hemispheres of man: measurements with an image analyser. Neuropathol Appl Neurobiol. 1980;6:119-32.

18. Seshadri S, Wolf PA, Beiser A, et al. Stroke risk profile, brain volume, and cognitive function: the Framingham Offspring Study. Neurology. 2004;63:1591-9.

19. Jang JW, Park SY, Park YH, et al. A comprehensive visual rating scale of brain magnetic resonance imaging: application in elderly subjects with Alzheimer's disease, mild cognitive impairment, and normal cognition. J Alzheimers Dis. 2015;44:1023-34.

20. Lebrão ML, Laurenti R. Saúde, bem-estar e envelhecimento: o estudo SABE no Município de São Paulo. Rev Bras Epidemiol. 2005;8:127-41.

21. Dourado JC, Pereira JL, Albuquerque LAF, et al. Indices of agreement between neurosurgeons and a radiologist in interpreting tomography scans in an emergency department. Arq Neuropsiquiatr. 2015;73:688-91. 